IJBPAS, December, Special Issue, 2021, 10(12): 287-296

ISSN: 2277-4998

International Journal of Bhology, Pharmacy and Allied Sciences (IJBPAS)

'A Bridge Betueen Caboratory and QRender'

www.ibpas.com

\title{
A STUDY OF CORD LIPID PROFILE, BLOOD SUGAR IN TERM AND PRETERM NEWBORNS
}

\section{OM PRAKASH JHA ${ }^{1^{*}}$, JAY PRAKASH JHA ${ }^{2}$, RUCHIRA DUBEY ${ }^{3}$, PRADEEP KUMAR $^{4}$, PREETI SHARMA $^{5}$ AND TK MOHAPATRA ${ }^{6}$}

1: Research Scholar, Department of Biochemistry Santosh Medical College and Hospital, Santosh Deemed to be University, Ghaziabad, UP, India

2: Research Scholar, Department of Biochemistry Indra Gandhi Institute of Medical Sciences Patna, Bihar India

3: Tutor, Department of Biochemistry Venkateshwara Institute of Medical Science, Gajraula, UP, India

4: Department of Biochemistry, Autonomous State Government Medical College, Fatehpur,

$$
\text { UP, Indi }
$$

5: Associate Professor, Department of Biochemistry, Santosh Medical College and Hospital, Santosh Deemed to be University, Ghaziabad, UP, India

6: Former Professor \& HOD, Department of Biochemistry, Santosh Deemed to be

$$
\text { University, Ghaziabad, UP, India }
$$

*Corresponding Author: Om Prakash Jha; E Mail: om2prakashjha@gmail.com

Received $19^{\text {th }}$ Aug. 2021; Revised 20 ${ }^{\text {th }}$ Sept. 2021; Accepted 29 ${ }^{\text {th }}$ Oct. 2021; Available online $1^{\text {st }}$ Dec. 2021

\section{https://doi.org/10.31032/IJBPAS/2021/10.12.2029}

\begin{abstract}
Background: Diabetic mellitus jaundice, and cardiovascular problems are the leading causes of death in both developed and developing countries. Preterm newborns with higher levels of cord blood glucose, total cholesterol, triglyceride, LDL, and VLDL may have a higher future risk of cardiovascular disease in diabetes mellitus. Early detection, dietary changes, and adequate
\end{abstract}


management may be able to correct risk factors and avoid disease in the future. The goal of this study is to do biochemical profiling in preterm and full-term neonates' umbilical cord blood.

Methods: It is retrospective and observational study conducted for a period of two years from October 2018 to November 2020 in the Departments of Biochemistry and Gynecology of Santosh Medical College and Hospitals, Ghaziabad and K. D Medical College and Research center Mathura.

Results: Umbilical Cord blood serum blood sugar, total cholesterol, triglyceride, LDL, and VLDL were higher in preterm babies compared to term babies in a study of 200 neonates, with 100 (50 percent) term and 100 (50 percent) preterm babies. Baby weight was also significantly increased $(<0.05)$ in term babies compared to preterm babies.

Conclusion: There is an inverse association between gestational age and several biochemical markers, and the preterm group's abnormal biochemical profile may be a risk factor for the development of diabetes and cardiovascular disease later in life.

\section{Keywords: Newborn, Preterm neonates, Umbilical cord blood study, Low birth weight INTRODUCTION}

The nutritional status of a foetal life impacts the quality of future adult life, and thus determines the wellbeing of a baby. The foetus is known to be the reference source for establishing normal nutritional concentrations in blood and tissue (1). Atherosclerosis, a leading cause of cardiovascular disease, is a silent process that begins in childhood and continues for decades. Epidemiological studies show that serum cholesterol and coronary heart disease have a strong independent connection. Early artherogenesis and a significant rise in fatty streaks in human babies have been linked to maternal hypocholesterolemia. The nutritional state of the foetus can be measured in the cord blood serum in terms of glucose, protein, and lipids. Cord blood can also be used to screen for a variety of metabolic illnesses. Cord blood glucose and blood glucose at 4-6 hours of age (before the second meal) should be assessed for hypoglycemia screening, according to Hawdon \& Ward Platt (1993) (2). Anderson et al. (1993) found that throughout the first 50 years of life, 38 percent of uncomplicated term infants born in Kathmandu, Nepal had a blood glucose content of 2.6mmol/lit (3). 107 cases of asymptomatic or symptomatic newborn hypoglycemia were evaluated with neurodevelopmental outcome in an Indian follow-up neonatal hypoglycemic research 
(4). Low birth weight (LBW) and foetal malnutrition are risk factors for the adult stages of lipid disorders, myocardial ischemia, hypertension, obesity, and diabetes $(5,6)$.

Disorders in lipid metabolism, as well as high levels of LDL-C, total cholesterol (TC), and apolipoproteinB (ApoB) in young adults, have been linked to cardiovascular disease later in life(7).Levels of ApoB, ApoB/apoA1 ratio, and LDL-C concentrations are sensitive predictors for later coronary heart disease in infants, according to Dirisamer et al (2006) (8). The ApoB/ApoA-1 ratio, also known as the atherogenic index, is a sensitive marker for monitoring coronary artery disease that has been shown to be actively monitored during the first year of life $(9,10)$. The capacity to appropriately quantify CAD risk may be considerably improved by screening patients for ApoA-1 and ApoB levels (11). Containing Low birth weight is linked to an increased risk of cardiovascular disease, hypertension, and type 2 diabetes (12). In low birth weight neonates with relative insulin resistance, changes in blood lipids can raise the risk of CVD in adulthood. At the age of puberty, LBW has the same risk of subsequent atherosclerotic illnesses as smoking or hypertension (13). As a result, it appears that there is a link between birth weight and adult CVD mortality. As a result, the current study was designed to look into the relationship between lipid characteristics and neonatal birth weight.

Because an unbalanced lipid profile might persist into adulthood, it's important to identify at-risk children during the prenatal and postnatal periods, and to pay extra attention to them in terms of lifestyle change to prevent future issues, notably cardiovascular complications. The lipid profile of umbilical cord blood can be used to perform this type of screening. The goal of this study is to examine and determine all lipid characteristics in umbilical cord blood, as well as to correlate pre-term and full-term infant levels. Is there a link between birth weight and foetal gender and lipid profiles in neonate.

\section{MATERIALS AND METHODS}

This study on umbilical cord blood serum was conducted at Biochemistry and Gynecology of Santosh Medical College and Hospitals, Ghaziabad, and K D Medical College Hospital and Research Center. The study had a sample size of 200 participants, divided into two groups: control and experimental. This study enlisted the participation of 200 newborns. The neonates were divided into two groups. 100 preterm newborns (31 to 36 weeks) were in group A, 
whereas 100 full-term neonates were in group B. (from 37 weeks to 41 weeks). A computerized electronic weighing scale was used to estimate the birth weight.

The study's participants were chosen at random. Throughout all stages of labour, the clinical circumstances of the full-term and preterm delivery individuals were closely monitored. About $5 \mathrm{ml}$ of human umbilical cord blood was taken from the placental end of the umbilical vein in plain and fluoride vials. Total cholesterol (TC), triglycerides (TG), HDL cholesterol (HDL-C), VLDL, and LDL-C, as well as blood glucose, were measured in serum by centrifugation and analysed the same day.

Biochemical indicators were measured using a Beckmancoulter-AU480 Fully Automatic Analyzer. A fully automatic-analyzerBeckmancoulter-AU480 was used to measure cord blood glucose, total cholesterol, TG, HDL, LDL and VLDL.

\section{Inclusion criteria}

Gestational age between 31 to 41 weeks included in this study.

\section{Exclusion criteria}

The expectant moms who were eligible for this trial were between the ages of 20 and 35 and had no signs of septicemia, other hematological infections, or problems, as well as being negative for VDRL, human immunodeficiency, hepatitis B, or hepatitis C viruses. This study did not include pregnant women with diabetes, hypertension, or chronic illnesses.

Statistical analysis

SPSS software was used to examine the data. The students' test was utilized to compare the study groups. The $\mathrm{p}$ value that was significant was $<0.05$.

\section{RESULTS}

Table 1: Compression Biochemical parameters Pre-term and Full-Term

\begin{tabular}{|c|c|c|c|}
\hline \multirow{2}{*}{$\begin{array}{c}\text { BIOCHEMICAL } \\
\text { PARAMETERS }\end{array}$} & Pre-term & Full- Term & \multirow{2}{*}{ P-Value } \\
\cline { 2 - 4 } & Mean \pm SD & Mean \pm SD & \\
\hline Baby weight (kg) & $\mathbf{1 . 5 0} \pm \mathbf{0 . 2 3}$ & $\mathbf{3 . 0 5} \pm \mathbf{0 . 2 8}$ & $\mathbf{0 . 0 0 1}$ \\
\hline Blood Sugar & $\mathbf{4 5 . 5 8 \pm 1 1 . 6 5}$ & $\mathbf{8 1 . 7 6} \pm \mathbf{5 . 2 9}$ & $\mathbf{0 . 0 0 1}$ \\
\hline Total cholesterol & $\mathbf{7 1 . 2 8} \pm \mathbf{0 . 7 2}$ & $\mathbf{6 5 . 0 6} \pm \mathbf{0 . 6 8}$ & $\mathbf{0 . 0 0 0}$ \\
\hline Triglyceride & $\mathbf{4 8 . 7 8 \pm 0 . 6 8}$ & $\mathbf{5 6 . 1 6} \pm \mathbf{0 . 4 9}$ & $\mathbf{0 . 0 0 4}$ \\
\hline HDL & $\mathbf{3 0 . 1 6} \pm \mathbf{0 . 4 2}$ & $\mathbf{3 0 . 0 5} \pm \mathbf{0 . 4 5}$ & $\mathbf{0 . 9 1 7}$ \\
\hline LDL & $\mathbf{3 1 . 3 6} \pm \mathbf{0 . 7 7}$ & $\mathbf{2 5 . 9 0 \pm 0 . 8 2}$ & $\mathbf{0 . 0 0 0}$ \\
\hline VLDL & $\mathbf{9 . 5 0} \pm \mathbf{0 . 1 8}$ & $\mathbf{1 0 . 1 1} \pm \mathbf{0 . 1 2}$ & $\mathbf{0 . 0 0 4}$ \\
\hline
\end{tabular}

The mean value of baby weight in preterm neonates was $1.50 \pm 0.23$ and term neonates were $3.05 \pm 0.28$

The mean value of serum blood sugar in preterm neonates was $45.58 \pm 11.65$ and term neonate's value was $81.76 \pm 5.29$. The mean value of serum total cholesterol in term and 
preterm neonates was found to be $65.06 \pm 0.68$ and $71.28 \pm 0.72$ respectively. Significant lower values of serum total cholesterol were observed in term neonates as compared to preterm neonates (P. value 0.000$)$

The mean value of serum triglycerides in and term neonates was found to be $56.16 \pm 0.49$ and $48.78 \pm 0.68$ respectively. Significant lower values of serum triglycerides were observed in term neonates as compared to preterm neonates $(\mathrm{p}<0.04)$.

The mean value of serum high density lipoprotein cholesterol (HDL) in term and preterm neonates was found to be $30.05 \pm$ 0.45 and $30.16 \pm 0.45$ respectively. No significant difference was observed in HDL cholesterol vales in term neonates as compared to preterm neonates. The mean value of serum low density lipoprotein cholesterol (LDL) in term and term neonates was found to be $25.90 \pm 0.82$ and $31.36 \pm$ 0.77 respectively. Significant higher values of serum LDL-cholesterol were observed in term neonates as compared to preterm neonates $(p<0.000)$. The mean value of serum very low density lipoprotein cholesterol (VLDL) in term and term neonates was found to be $10.11 \pm 0.12$ and $9.50 \pm 0.18$ respectively. Significant lower values of serum VLDL-cholesterol were observed in term neonates as compared to preterm neonates $(\mathrm{p}<0.01)$.

\section{DISCUSSION:}

Cord blood biochemical markers are appealing to use as outcome measures showing the newborn's status because of their objective nature, which reflects foetal anaerobic metabolism and eventual development of metabolic disease.

Low birth weight is a big issue in India, where the frequency of low birth infants is as high as $28 \%$. Neonatal birth weight is a good predictor of the mother's health and nutritional state, as well as the neonates' survival growth, long-term health, and psychosocial development. LBW neonates, weighing less than $2.5 \mathrm{~kg}$, have a significant mortality rate and are the primary contributor to neonatal deaths. Those who survive infant and under-five mortality have reduced immune function and are at a high risk of developing adult-onset disease early in life.

Because most foetal lipids are produced denovo through glucose conversion to diverse fatty acid-containing molecules, the lipid profile of the umbilical cord reflects lipid metabolism during foetal development and at birth. Only a little portion of it comes from placental circulation. Total cholesterol levels rise after birth, therefore it's possible that preterm neonates' total cholesterol levels are 
equivalent to or lower than those of term infants. Total cholesterol levels in human umbilical cord blood were discovered to be lower than those observed in adult plasma.

Total cholesterol levels in human umbilical cord blood were discovered to be lower than those observed in adult plasma. This was agreed upon by Hellmuth (14). However, our findings showed that the premature group's cholesterol levels were much greater than those of the term group, which was consistent with a prior study (15).

The study included 100 preterm 31 to 36 week neonates and 100 full-terms 37 to 41 week neonates out of a total of 200 participants. The highest concentration of cord blood cholesterol was seen in term neonates. Preterm infants had the greatest levels of cholesterol in their cord blood. There was a significantly significant difference in the mean triglyceride levels throughout the entire study group.

Furthermore, the decrease of specific metabolites such as glucose in blood obtained by cordcentesis in fetuses with IUGR has been described, considering that glucose is the main substrate for fetal energy metabolism and its demand increases as growth progresses (16). This fact could be the result of alterations in the placenta or in fetal glucose metabolism $(17,18)$.
The mean cord blood glucose level with SD in preterm neonates was $45.58 \pm 11.65 \mathrm{mg} / \mathrm{dl}$, while term neonates had $81.76 \pm 5.29 \mathrm{mg} / \mathrm{dl}$, according to the study. This average value was similar to the umbilical cord blood glucose level $(3.9 \pm 0.5 \mathrm{mmol} / \mathrm{L}$ or $70 \mathrm{mg} / \mathrm{dL})$ found in one of the studies(19). The study also showed that the mode of cord blood glucose level remains unchanged throughout caesarean and regular deliveries. The mean cholesterol levels were preterm $71.28 \pm 0.72$ $\mathrm{mg} / \mathrm{dl}$ and full- term $81.76 \pm 5.29$ which is less than the reports of Puspedra et al, 2013 and Triglyceride where the values were 48.78 \pm 28 and full term 56.16 \pm 0.4910 and HDL levels were preterm $30.16 \pm 0.42$,full-term values $30.05 \pm 0.45$. The values were higher ranging from $64 \pm 19$ has been noted in other studies $(20,21,22)$. The mean triglyceride levels noted in present study were much lower than the adult values. Perhaps the quiescent state of fat utilization in the fetus and the absence of need for fat mobilization are responsible for low serum triglyceride at birth. The values of HDL-C and LDL-C were similar to those reported in other studies (23). According to reports, the stresses of labour or surgery, as well as the use of anesthetics, have no effect on the glucose levels in the umbilical cord blood. However, using a bigger sample size in the study would aid in 
scientifically explaining the aetiology of the findings.

Another study indicated that all values in the cord blood lipid profile were lower in preterm newborns compared to term neonates, however there was a statistically significant difference in TC levels $(\mathrm{P}<0.001)$ and no statistically significant change in HDL or LDH levels (24). Higher cord blood lipid profile levels in preterm neonates were found in our study, which could be explained by the fact that preterm newborns lack both hepatic carbohydrate and subcutaneous adipose stores, resulting in low circulatory fuel and the possibility of running out (25). The elevation in cord blood lipid levels could be due to a metabolic response to give enough energy to organs such as the brain (26).

\section{CONCLUSIONS}

Our findings show that TC, LDLC, and HDL levels were considerably higher in preterm neonates than in term newborns, indicating a shift toward a shoddier lipid profile in preterm neonates. Future research is required to answer unanswered concerns such as whether the atherogenic index of preterm/full-term neonates affects body metabolism and whether it raises the risk of cardiovascular disease in fully-grown/adult life.

\section{ACKNOWLEDGEMENT}

Authors would like to thank the management of Santosh Deemed to be University Ghaziabad.

\section{REFERENCES}

[1] American Academy Pediatrics Committee on Nutrition- Nutritional needs of Preterm Infants. In: Kleinman RE, ed. Pediatric Nutrition Handbook. $6^{\text {th }}$ edition. Elk Grove Village,IL: American Academy Pediatrics; 2009: 79-112.

[2] Rajasekhar D, Saibaba KSS, SrinivasaRao PVLN, Latheef SAA, Subramanyam G. Lipoprotein (a): Better assessor of coronary heart disease risk in South Indian population. Ind $\mathrm{J}$ Clin Biochem. 2004; 19: 53-9. [PMC free article] [PubMed] [Google Scholar]

[3] Hawdon JM, Ward Platt MP. Metabolic adaptation in small for gestational age infants. Arch. Dis. Childh. 1993; 68: 262- 268.

[4] Anderson S, Shakya KN, Shrestha LN, de L Costello AM. Hypoglycaemia: A common problem among uncomplicated newborn infants in Nepal. Journal Tropical Pediatr. 1993; 39: 273-277. 
[5] Singh M, Singhal PK, Paul VK, Deorari AK, Sundaram KR, Ghorpade MD, et al. Neurodevelopmental outcome of asymptomatic and symptomatic babies with neonatal hypoglycaemia. Indian J Med Res. [B]. 1991; 94: 610.

[6] Gale CR, Ashurst HE, Hall NF, MacCallum PK, Martyn CN. Size at birth and carotid atherosclerosis in later life. Atherosclerosis. 2002; 1:141-147.

[7] Godfrey KM, Barker DJ: Fetal nutrition and adult disease. Am $\mathrm{J}$ ClinNutr. 2000; Suppl. 5: 1344S$1352 \mathrm{~S}$.

[8] Abdul -Rahim HF, Hussein A, Bjertness E, Giecaman R, Gordon $\mathrm{NH}$, Jervell J. The metabolic syndrome in the west bank population. An urban - rural Comparison. Diabetic care 2001; 24: 275-279.

[9] Dirisamer A, stadler A, Bucek RA, Widhalm K. Apo B - 100 and ApoB/Apo-A-1 ratio in children and adolescents from families with very early myocardial infarction. Actapaediatr 2006; 95: 810-3.
[10] Dolphin PJ. Breckenridge WC, Dolphin MA, Tan MH. The lipoproteins of human Umbilical cord blood, apolipoproteins and lipid levels. Atherosclerosis 1985; 51: 109-122.monalsschr, kinderheillkd, 2001 (suppl): 149-5256.

[11] Yavuz T, Yavuz O, Ozdemir I, Afsar Y. Cord blood lipoprotein profile after magnesium sulphate treatment in Pre - eclamptic patients. Actapaediatr 2006; 95: 1224-7.

[12] Sattar N, Williams K, Siriderman AD, Agostino RB, Haffner SM. Comparison of the associations of apolipoprotein B and high density lipoprotein cholesterol with other cardiovascular risk factors in patients with the metabolic syndrome in the insulin resistance Atherosclerosis study. Circulation 2004; 110: 2687-93.

[13] Baker DJ, Hales CN, FallCH, Osmond C, Phipps K, Clark PM. Type 2 (non-insulin dependent) diabetes mellitus, hypertension and hyperlipidemia (syndrome): relation to reduced fetal growth. Diabetolgia. 1993; 36: 62-67. 
[14] MJ Law C, Zhang KL, Osmond C, Stein C, Barker D. Effects of infants birth weight and maternal body mass index in pregnancy on components of the insulin resistance syndrome in China. Ann Intern Med 2000; 132: 253-260.

[15] Hellmuth K.: Specificity of placental opzime for interforametric diagnosis Kline Wchneschr. 1926; 5: 2006.

[16] Pushpendramagon, RS bharatwaj, Manorama Verma, Jugesh Chatwal. Cord blood lipid profile at birth among normal Indian newborns and its relation to gestational maturity and birth weight- A cross sectional study. Indian journal of research. July 2013; 2(7): 215-218.

[17] Barnes K, Westael PJ, Payne E.S. Neonatal plasma lipids. Medical Journal of Australia. 1972; 28: 1002.

[18] Dubowitz LMS, Dubovitz V, Goldberg C. Clinical assessment of gestational age in the newborn infant. Journal of pediatrics 1970; 77(1): 1-10.

[19] Jungner I, Sniderman AD, Furberg C, Aastvert AH, Holme I, Walldius G. Does low- density lipoprotein size add to atherogenic particle number in predicting the risk of fetal myocardial infarction? Am J Cardiol 2006; 97: 943-946.

[20] Diaz M., Leal C., Ramon y. Cajal J: Jiminez MD, Martinez H., Pocovi M., Grande F: Cord blood lipoprotein-cholesterol: relationship birth weight and gestational age of newborns. Metabolism, 1989; 38: $435-438$.

[21] Van der Schouw YT, AL MD, Hornstra G, Bulstra-ramakers MT, Huisjes HJ. Fattyacid composition of serum lipids of mothers and their babies after normal and hypertensivepregnancies.

Prostaglandins, leukotrienes and essential fatty acid 1991 dec1; 44(4): 247-52.

[22] Kherkeulidze P, Johansson J, Carlson LA.High density lipoprotein size

distribution incordblood.ActaPaediatrica. 1991 Aug; 80 (8-9):770-9.

[23] Averna MR, Barbagallo CM, Di GP, Labisi M, Pinna G, Marino G, et al Total cholesterol, LDL- cholesterol and apoprotein B in Umbilical cord blood: cross- sectional study. Minerva Pediatr. 1992 Sep; 44(9): 395-9 
[24] L. M. Molina and R. Hernandez Barbosa, Intrauterine growth retardation (IUGR) and biochemical alterations, NOVA 3 (2005) 88-94.

[25] V. Karsdop and J. Van, Amino acids, glucose and lactate concentration in umbilical cord blood in relation toumbilical artery flow patterns, Eur J ObstetGynecol 57 (1994) 117-122.

[26] U. Nicolini, P. Nicolaides, N. Fisk, J. Vaughn, L. Fusi and R. Glesson et al., Limited role of fetal blood sample in prediction of outcome in intrauterine growth retardation, Lancet 336 (1990) 768-772. 